\title{
Mitochondrial translocation and interaction of cofilin and Drp1 are required for erucin-induced mitochondrial fission and apoptosis
}

\author{
Guobing Li ${ }^{1, *}$, Jing Zhou ${ }^{1, *}$, Amit Budhraja ${ }^{3}$, Xiaoye Hu${ }^{1}$, Yibiao Chen ${ }^{1}$, Qi Cheng ${ }^{1}$, \\ Lei Liu ${ }^{1}$, Ting Zhou ${ }^{1}$, Ping $\mathbf{L i}^{2}$, Ehu Liu ${ }^{2}$, Ning Gao ${ }^{1}$ \\ ${ }^{1}$ College of Pharmacy, 3rd Military Medical University, Chongqing 400038, China \\ ${ }^{2}$ State Key Laboratory of Natural Medicines, China Pharmaceutical University, Nanjing 210009, China \\ ${ }^{3}$ Cell \& Molecular Biology, St. Jude Children's Research Hospital, Memphis TN 38105, USA \\ *These authors have contributed equally to this work
}

Correspondence to:

Ning Gao, e-mail: gaoning59@163.com

Ehu Liu, e-mail: liuehu2011@163.com

Keywords: Cofilin, Drpl, mitochondrial fission, erucin, apoptosis

Received: September 18, $2014 \quad$ Accepted: November 20, $2014 \quad$ Published: December 16, 2014

\section{ABSTRACT}

Cofilin is a member of the actin-depolymerizing factor (ADF) family protein, which plays an essential role in regulation of the mitochondrial apoptosis. It remains unclear how cofilin regulates the mitochondrial apoptosis. Here, we report for the first time that natural compound 4-methylthiobutyl isothiocyanate (erucin) found in consumable cruciferous vegetables induces mitochondrial fission and apoptosis in human breast cancer cells through the mitochondrial translocation of cofilin. Importantly, cofilin regulates erucin-induced mitochondrial fission by interacting with dynamin-related protein (Drp1). Knockdown of cofilin or Drp1 markedly reduced erucin-mediated mitochondrial translocation and interaction of cofilin and Drp1, mitochondrial fission, and apoptosis. Only dephosphorylated cofilin (Ser 3) and Drp1 (Ser 637) are translocated to the mitochondria. Cofilin S3E and Drp1 S637D mutants, which mimick the phosphorylated forms, suppressed mitochondrial translocation, fission, and apoptosis. Moreover, both dephosphorylation and mitochondrial translocation of cofilin and Drp1 are dependent on ROCK1 activation. In vivo findings confirmed that erucin-mediated inhibition of tumor growth in a breast cancer cell xenograft mouse model is associated with the mitochondrial translocation of cofilin and Drp1, fission and apoptosis. Our study reveals a novel role of cofilin in regulation of mitochondrial fission and suggests erucin as a potential drug for treatment of breast cancer.

\section{INTRODUCTION}

Mitochondria are double membrane-bound organelles found in most eukaryotic cells, where they play essential and diverse roles in cellular physiology, including growth, division, energy metabolism, and apoptosis [1-3]. Mitochondrial morphology is regulated by the balance of two continuous antagonistic processes: fusion and fission [4]. Under physiological conditions, mitochondria are elongated and filamentous, but they undergo extensive fragmentation during apoptosis [5]. Apoptotic fission is associated with remodeling of the cristae, which is characterized by the opening of their tubular junction. This process results in the complete release of proapoptotic factors, such as cytochrome c, which is required in the cytosol for the activation of downstream effector caspases [6]. A number of evidence revealed that dynamin-related protein 1 (Drp1) participates in mitochondrial fission. Drp1 is a GTPase that causes scission of the mitochondrial outer membrane, resulting in fission of mitochondrial tubules into fragments [7, 8]. Drp1 translocates from the cytosol to the mitochondria and mediates mitochondrial fission prior to caspase activation and apoptosis [9]. Drp1 is responsible for cytochrome $\mathrm{c}$ release and caspase 
activation [10]. However, the mechanism by which Drp1 is recruited to mitochondria remains unresolved.

Cofilin is a member of the ADF/cofilin family of small actin-binding proteins found in all eukaryotic cells, and it regulates actin dynamics by increasing the rate of actin depolymerization and facilitating actin filament turnover [11]. Cofilin plays an essential role in regulation of the mitochondrial apoptosis [12]. Recent findings indicate that after induction of apoptosis, cofilin translocates from the cytosol to the mitochondria prior to the release of cytochrome $\mathrm{c}[12,13]$. Knockdown of cofilin results in the inhibition of both cytochrome c release and of apoptosis [12]. In addition, recent evidence has implicated cofilin in regulation of mitochondrial dynamics and functions [13]. Cofilin functions as a biosensor that integrates cytoskeletal regulation with mitochondrial function. The results of another study indicated that under certain cellular conditions cofilin might directly or indirectly associate with mitochondria and modulate mitochondrial functions [14]. However, the mechanism by which cofilin affects mitochondrial dynamics and functions, and is responsible for mitochondrial apoptosis remains unknown.

Rho-associated coiled coil-containing protein kinase 1 (ROCK1) belongs to a family of serine/threonine kinases that are activated by Rho GTPases or caspase-3 via cleavage the C-terminal auto-inhibitory domain away from the kinase active site $[15,16]$. Recent studies have shown that ROCK1 plays an important role in regulation of apoptosis in various cell types and animal disease models [16-18]. It has been shown that ROCK1 plays a critical role in mitochondrial fission, which results in recruitment of dynamin-related protein-1 (Drp1) to the mitochondria [19]. ROCK1 has also been shown to play a critical role in dephosphorylation and mitochondrial translocation of cofilin [17]. However, the precise mechanism by which ROCK1 regulates the phosphorylation status of Drpl and cofilin during mitochondrial fission and apoptosis remains unclear.

The natural compound 4-methylthiobutyl isothiocyanate (erucin) is found in consumable cruciferous vegetables and possesses chemopreventive and chemotherapeutic activities [20]. Increasing evidence suggests that erucin exerts its antiproliferative effects by inducing cell cycle arrest and apoptosis in various cancer cell lines in vitro [21,22] and in tumor xenograft models in vivo [23]. The results of recent studies suggest that a mitochondrion-dependent pathway may play an important role in erucin-mediated apoptosis [24]. However, the molecular mechanisms by which erucin regulates the mitochondrial apoptosis pathway in human breast cancer cells has not yet been explored. Here, we report for the first time that erucin potently induced mitochondrial fission and apoptosis through mitochondrial translocation and interaction of cofilin and Drp1. Importantly, Rho-associated coiled coil-containing protein kinase 1 (ROCK1) was found to play an important role in regulating the dephosphorylation of cofilin and Drp1. Our in vivo findings indicated that the erucin-mediated inhibitory effects on tumor growth in a
MDA-MB-231 xenograft mouse model was also associated with dephosphorylation and mitochondrial translocation of cofilin and Drp1, mitochondrial fission, and apoptosis. These findings provide a novel mechanistic basis for the application of erucin in the treatment of breast cancer.

\section{RESULTS}

\section{Erucin induces apoptosis and mitochondrial fission in human breast cancer cells}

First, we examined the effects of erucin on apoptosis and mitochondrial injury in human breast cancer MDAMB-231 and MCF-7 cells. Flow cytometry analysis revealed that exposure of MDA-MB-231 and MCF-7 cells to erucin resulted in a significant increase in mitochondrial injury (loss of $\nabla \Psi \mathrm{m}$ ) and apoptosis in dose- and timedependent manners (Fig. 1A and 1B). Consistent with these findings, the same erucin concentrations and exposure intervals caused cleavage and activation of caspase 9 and caspase 3 and degradation of PARP. These events were also accompanied by significant increases in the release of cytochrome $\mathrm{c}$ from the mitochondria into the cytosol (Fig. 1C and 1D). Immunofluorescence assay also revealed that cytochrome $\mathrm{c}$ was release from mitochondria to cytosol after erucin treatment (Fig. 1E and 1F).

Mitochondrial fission is related to the initiation of apoptosis [4, 12, 25], and therefore, we examined the effects of erucin on mitochondrial fission in both MDA-MB-231 and MCF-7 cells. Mitochondria were labeled by staining with the mitochondrion-selective probe Mitotracker Red CMXRos. Exposure of cells to erucin resulted in significant decreases in the average length of mitochondria (Fig. 1E and 1F). The electron microscopic studies revealed the increased mitochondrial fragmentation, as evidenced by a significant increase in small, punctate mitochondria in erucin-treated cells compared with control cells, which exhibited elongated filamentous mitochondria (Fig. $1 \mathrm{G}$ and $1 \mathrm{H}$ ). Taken together, these findings suggest that erucin induced mitochondrial fission, leading to the release of cytochrome c from mitochondria and cell death in human breast cancer cells.

\section{Erucin induces translocation of cofilin and Drp1 from the cytosol to mitochondria}

Recent evidence revealed that cofilin and Drp1 play critical roles in regulation of mitochondrial function by translocating from the cytosol to mitochondria $[14,17$, 26-28]. We next investigated whether mitochondrial translocation of cofilin and Drp1 is necessary for erucin to induce mitochondrial fission. Treatment of cells with erucin significantly increased the levels of cofilin and Drp1 in mitochondria and decreased cofilin and Drp1 levels in the cytosol in a time-dependent manner (Fig. 2A). Immunofluorescence microscopy was used to further detect the sub-cellular localization of cofilin and 
A

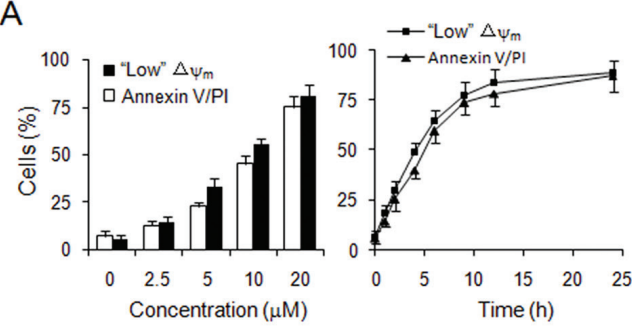

C

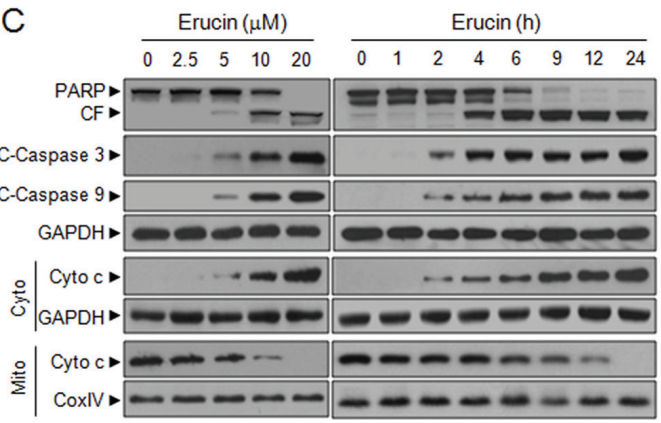

E
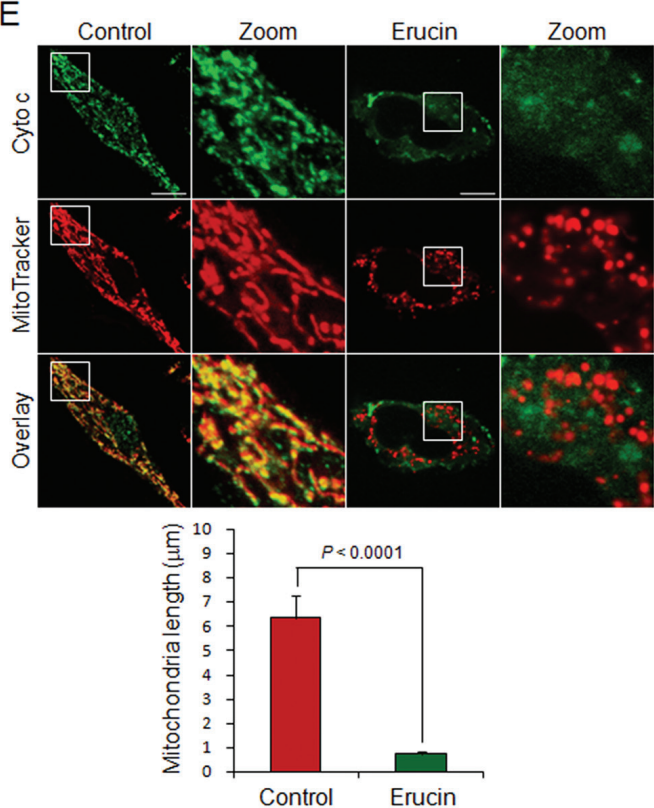

G

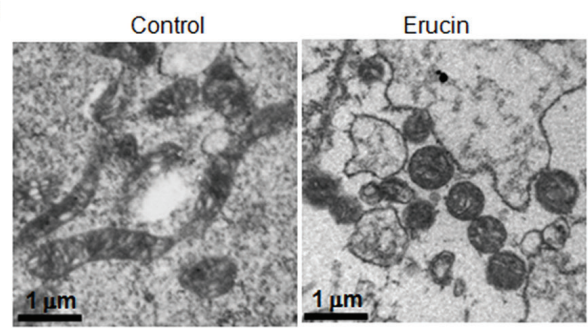

B
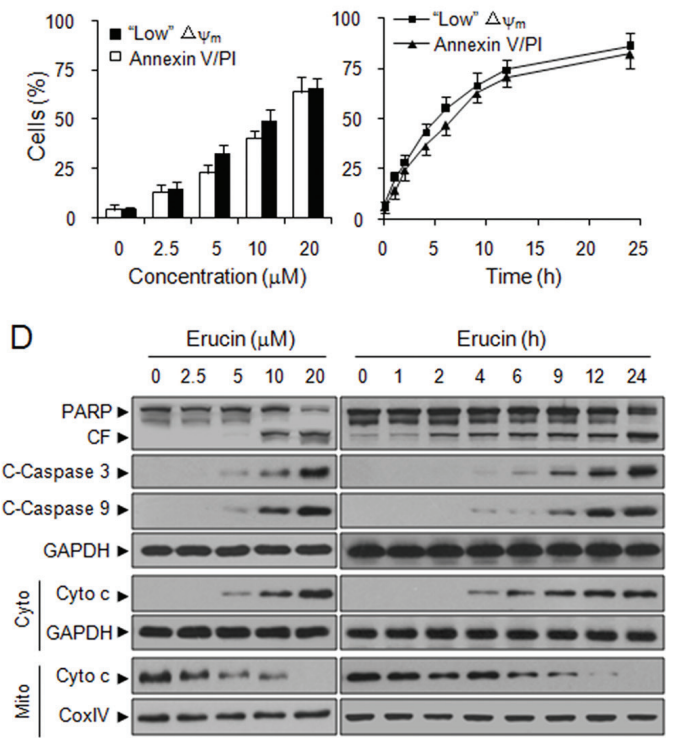

$\mathrm{F}$
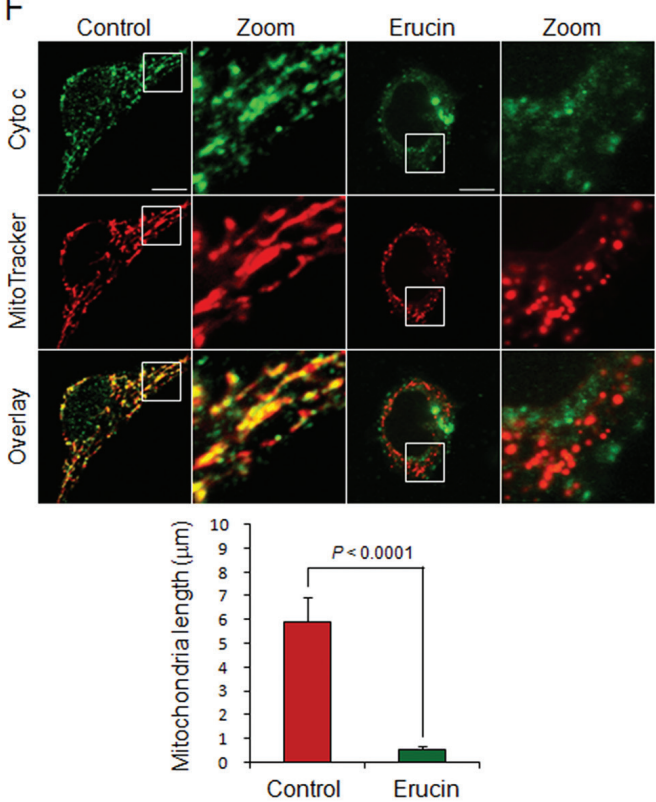

$\mathrm{H}$

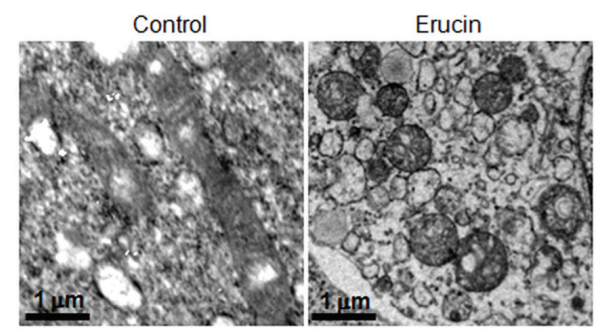

Figure 1: Erucin induces apoptosis and mitochondrial fission in human breast cancer cells. (A, B) MDA-MB-231 (A) and MCF-7 (B) cells were treated with various concentrations of erucin for $9 \mathrm{~h}$ or $20 \mu \mathrm{M}$ erucin for different time intervals as indicated. Apoptosis and loss of mitochondrial membrane potential $(\varangle \Psi \mathrm{m})$ were determined by flow cytometry. (C, D) Whole cell lysates, mitochondrial (Mito) and cytosolic (Cyto) fractions from MDA-MB-231 (C) and MCF-7 (D) cells were prepared and subjected to immunoblotting using antibodies against PARP, cleaved-caspase 3 (C-Caspase 3), cleaved-caspase 9 (C-Caspase 9), cytochrome c (Cyto c), GADPH and Cox IV. (E, F) MDAMB-231 (E) and MCF-7 (F) cells were treated with $20 \mu \mathrm{M}$ erucin for $6 \mathrm{~h}$, double-stained with Mitotracker Red CMXRos and cytochrome c (Alexa Fluor 488, green). Fluorescence images were collected by confocal microscopy. Scale bar represents $10 \mu \mathrm{m}$. Quantifications of mitochondrial length were performed as described in Methods. (G, H) MDA-MB-231 (G) and MCF-7 (H) mitochondrial morphology was evaluated by electron microscopy. Scale bar represents $1 \mu \mathrm{m}$. 


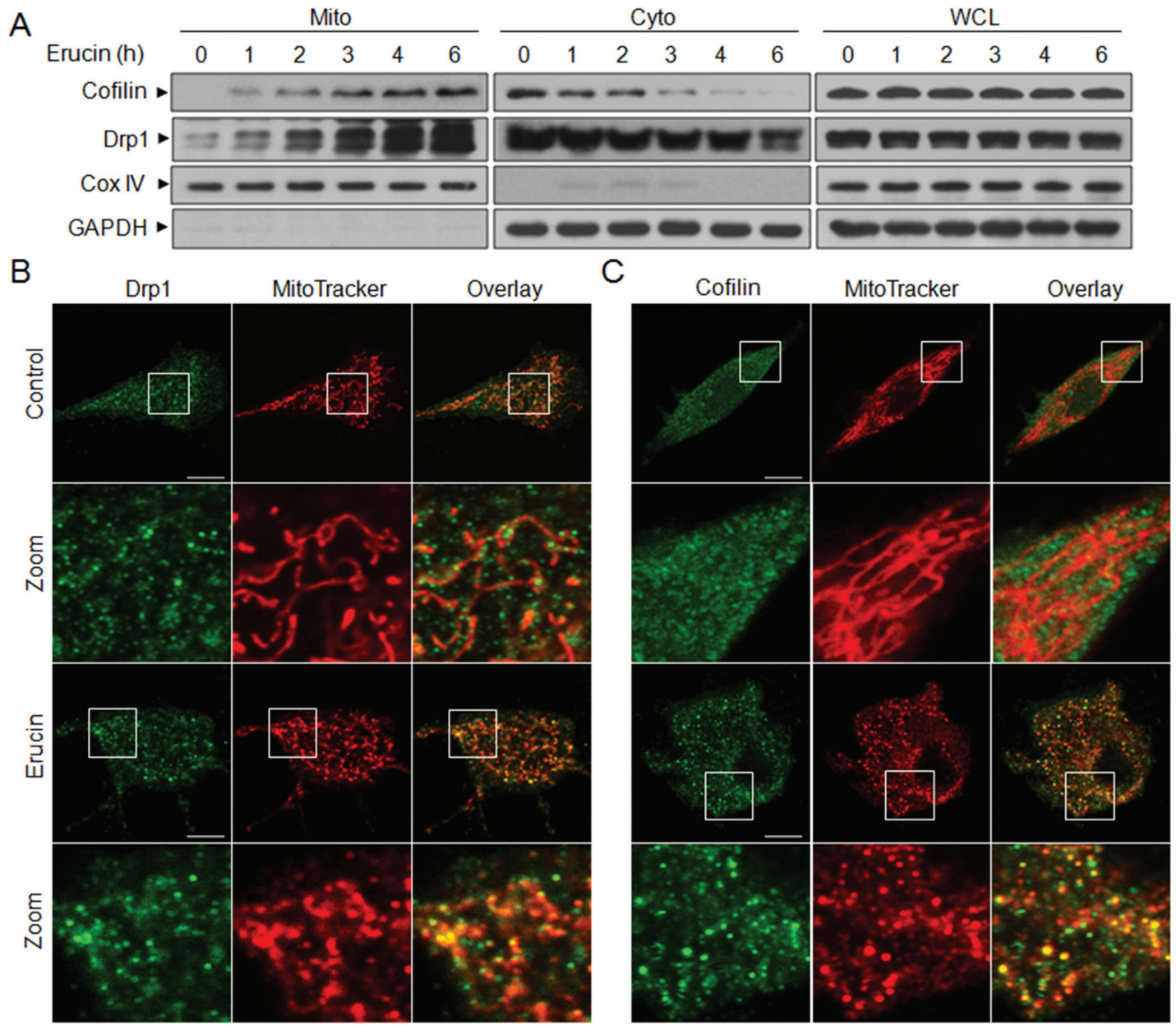

Figure 2: Erucin induces mitochondrial translocation of cofilin and Drp1. (A) MDA-MB-231 cells were treated with $20 \mu \mathrm{M}$ erucin, whole cell lysates (WCL), mitochondrial (Mito), and cytosolic (Cyto) fractions were prepared and subjected to Western blot analysis. (B, C) Cells were treated with $20 \mu \mathrm{M}$ erucin for $6 \mathrm{~h}$, stained with Mitotracker Red CMXRos and Drp1 (Alexa Fluor 647, green) or cofilin (Alexa Fluor 488, green). Fluorescence images were collected by confocal microscopy. Scale bar represents $10 \mu \mathrm{m}$.

Drp1 before and after erucin treatment. When cells were treated with erucin, cofilin and Drp1 signals were localized at the mitochondria (Fig. 2B and 2C). Mitochondrial fission was also observed following erucin treatment. These findings suggest that translocation of cofilin and Drp1 from the cytosol to the mitochondria is required for erucin-mediated mitochondrial fission.

\section{Erucin induces the interaction and colocalization of cofilin and Drp1 at the outer mitochondrial membrane}

To determine whether cofilin and Drp1 were localized at the outer mitochondrial membrane, mitochondrial fractions from MDA-MB-231 cells were digested with proteinase $\mathrm{K}$ as described previously [12], cofilin and Drp1 proteins were analyzed by Western blot analysis. Both mitochondrial cofilin and Drp1 induced by erucin were digested completely by proteinase $\mathrm{K}$, whereas Cyto c and CoxIV, which are internal mitochondrial markers are not digested by the protease treatment (Fig. 3A). These findings suggest that both cofilin and Drp1 are localized at the outer mitochondrial membrane after erucin treatment.

Because both cofilin and Drp1 participate in mitochondrial fission induced by erucin, we questioned whether cofilin could interact with Drp1 in response to erucin treatment in MDA-MB-231cells. Surprisingly, immunoprecipitation assay indicated that cofilin and Drp1 coimmunoprecipitated when cells were treated with erucin (Fig. 3B). Furthermore, immunofluorescence microscopic studies revealed a significant colocalization of cofilin and Drp1 in cells treated with erucin (Fig. 3C). Taken together, these findings indicate that the interaction and colocalization of cofilin and Drp1 at the outer mitochondrial membrane were essential for erucin-induced mitochondrial fission.

\section{Knockdown of cofilin decreases erucin-induced mitochondrial fission and apoptosis}

To further address the potential role of cofilin in erucin-mediated mitochondrial fission, a lentivirus shRNA 


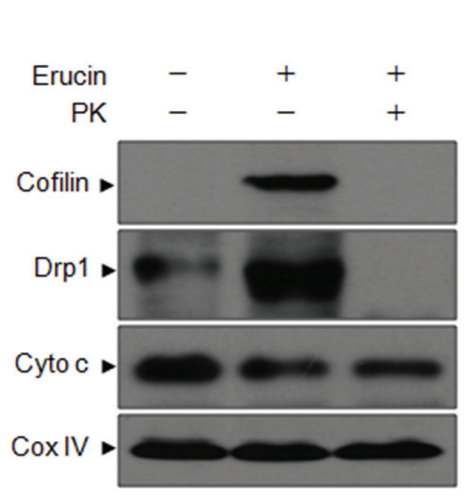

B
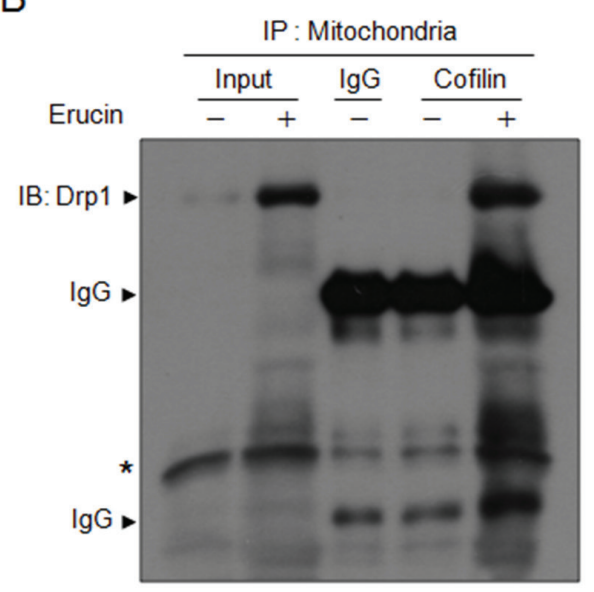

C
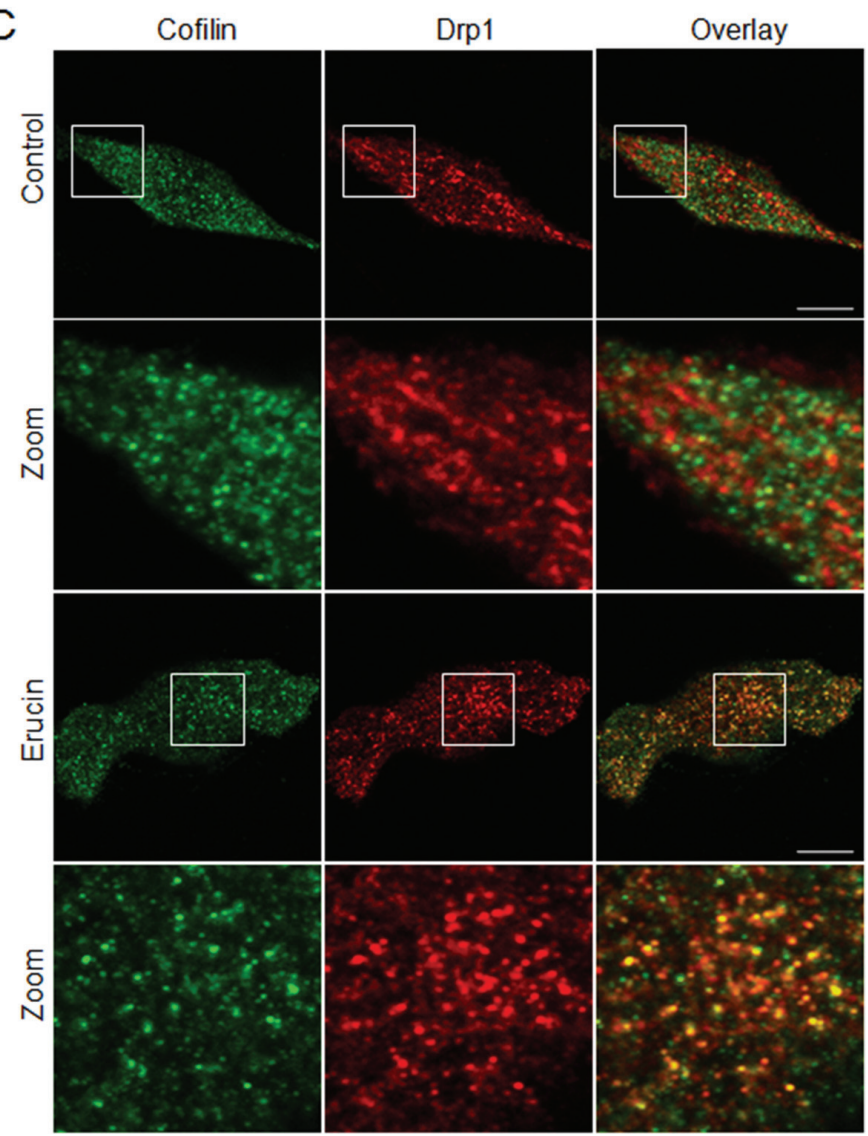

Figure 3: Erucin induces the interaction and colocalization of cofilin and Drp1 at the outer mitochondrial membrane. (A) MDA-MB-231 cells were treated with $20 \mu \mathrm{M}$ erucin for $6 \mathrm{~h}$, mitochondria were purified and incubated with $10 \mathrm{ng} / \mathrm{ml}$ proteinase $\mathrm{K}$ on ice for 10 minutes, then centrifuged at 17,000g for 15 minutes. After washing, pellets were lysed in $2 \%$ SDS buffer and determined by immunoblotting. (B) Mitochondrial fractions were prepared and subjected to immunoprecipitation using anti-cofilin antibody, and the associated Drp1 were determined using immunoblotting. IgG represents the heavy (approximately $57 \mathrm{kDa}$ ) or the light (approximately $25 \mathrm{kDa}$ ) chain of cofilin antibody. Asterisk represents nonspecific band. (C) Cells were treated with $20 \mu \mathrm{M}$ erucin for $6 \mathrm{~h}$, stained with cofilin (Alexa Fluor 488, green) and Drp1 (Alexa Fluor 647, red) and evaluated by confocal microscopy. Scale bar represents $10 \mu \mathrm{m}$.

approach was used to stably knockdown cofilin expression. Knockdown of cofilin markedly reduced erucin-mediated cofilin translocation from the cytosol to mitochondria (Fig. 4A). Immunoprecipitation of the mitochondrial fraction revealed that knockdown of cofilin markedly reduced the interaction between cofilin and Drp1 mediated by erucin (Fig. 4B). Knockdown of cofilin also reduced the colocalization between cofilin and Drp1 induced by erucin (Fig. 4C). Knockdown of cofilin significantly abrogated erucin-mediated mitochondrial fission and resulted in mitochondrial elongation (Fig. 4D and 4E). Moreover, depletion of cofilin significantly abrogated erucinmediated cytochrome $\mathrm{c}$ release, caspase 3 activation and apoptosis (Fig. 4F-4H).

\section{Knockdown of Drp1 decreases erucin-induced mitochondrial fission and apoptosis}

The translocation of Drp1 to mitochondria is a key event in mitochondrial fission $[9,26]$. We next tested whether specific suppression of Drp1 with lentivirus
shRNA affected erucin-induced mitochondrial fission and apoptosis. Infecting cells with Drp1 shRNA lentivirus significantly reduced Drp1 expression and erucinmediated Drp1 translocation from the cytosol to the mitochondria (Fig. 5A). Drp1 knockdown also markedly reduced the interaction and colocalization between cofilin and Drp1 mediated by erucin compared to that in control shRNA cells (Fig. 5B and 5C). Knockdown of Drp1 inhibited erucin-mediated mitochondrial fission and resulted in mitochondrial elongation (Fig. 5D and 5E). Moreover, Drp1 suppression by shRNA significantly abrogated erucin-mediated cytochrome $\mathrm{c}$ release, caspase 3 activation and apoptosis (Fig. 5F-5H).

\section{Dephosphorylation of cofilin (Ser 3) and Drp1 (Ser 637) is required for erucin-mediated mitochondrial fission and apoptosis}

Recent studies have indicated that only dephosphorylated cofilin is translocated to mitochondria during the initiation of apoptosis [12]. 


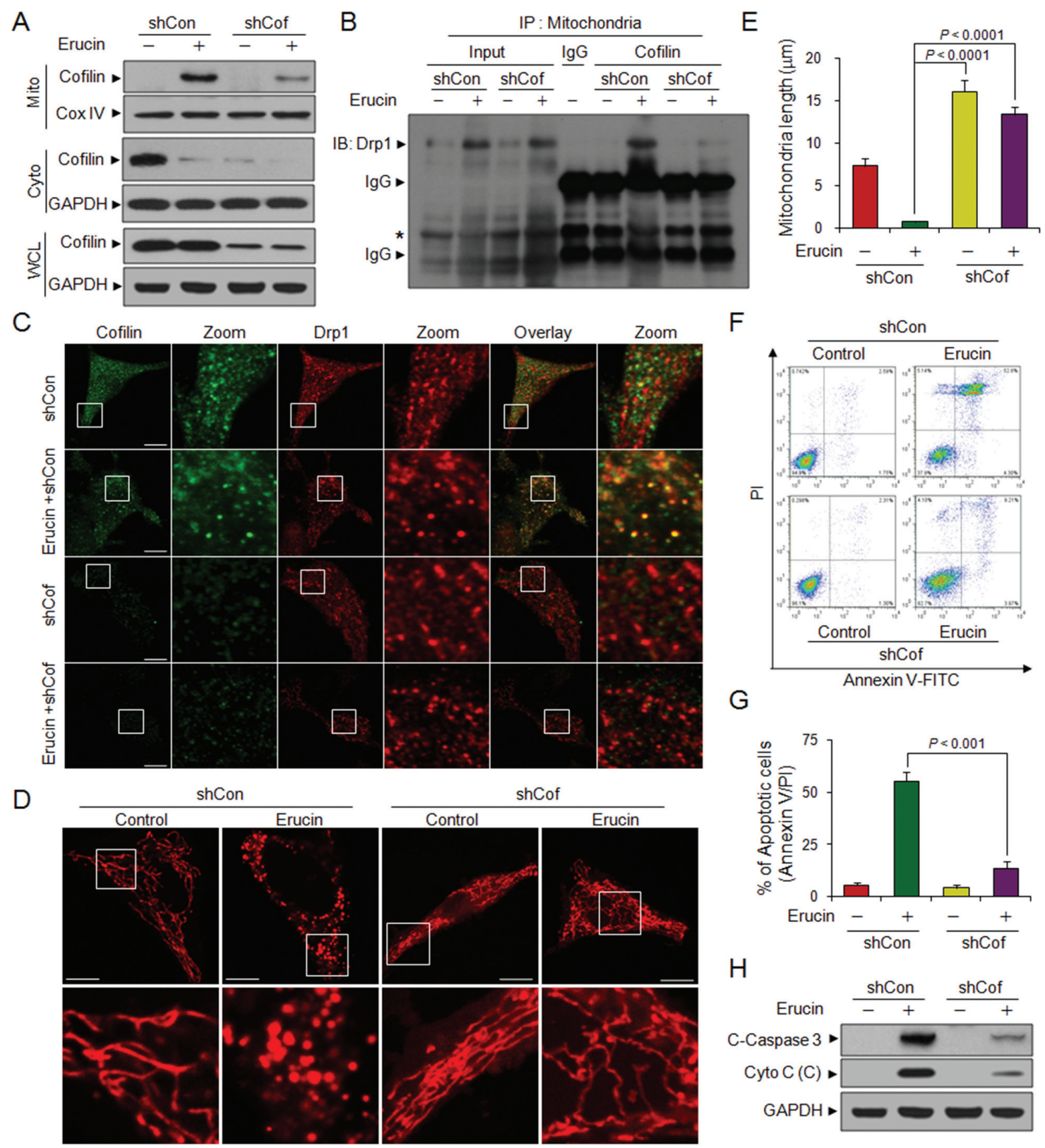

Figure 4: Cofilin knockdown decreases erucin-induced mitochondrial fission and apoptosis. MDA-MB-231 cells were infected with lentivirus containing constructs that encoded scrambled control shRNA (shCon) or human cofilin-specific shRNA (shCof). Stable cell lines were treated without or with $20 \mu \mathrm{M}$ erucin for $6 \mathrm{~h}$. (A) Whole cell lysates (WCL), mitochondrial (Mito), and cytosolic (Cyto) fractions were prepared and subjected to immunoblotting. (B) Mitochondrial fractions were prepared and subjected to immunoprecipitation to determine the interaction of cofilin and Drp1. Asterisk represents nonspecific band. (C) Cells were immunostained with cofilin (Alexa Fluor 488, green) and Drp1 (Alexa Fluor 647, red). (D, E) Cells were stained with Mitotracker Red CMXRos and observed by confocal microscope. Scale bar represents $10 \mu \mathrm{m}$. Mitochondrial length was measured as described in Methods. (F, G) Apoptosis was measured by flow cytometry. (H) C-Caspase 3 in whole cell lysates and Cyto c in cytosolic fractions (C) were determined by immunoblotting.

Next, we investigated whether erucin could affect the phosphorylation status of cofilin. Treating cells with erucin resulted in the dephosphorylation of cofilin (Ser 3) in the total cellular extract in a timedependent manner (Fig. 6A). To determine whether the phosphorylation status of cofilin could influence its ability to translocate to mitochondria and induce mitochondrial fission and apoptosis, two cofilin mutants mimicking either the dephosphorylated or phosphorylated forms were generated by changing Ser 3 to alanine (active; S3A) or glutamic acid (inactive; S3E) as described previously [29]. Overexpression of cofilin S3A enhanced, whereas cofilin S3E abolished, the mitochondrial translocation/ 


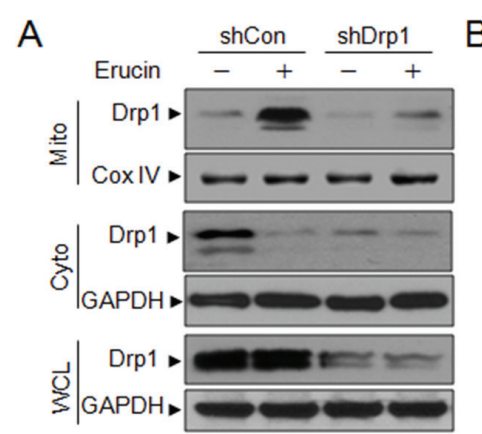

C

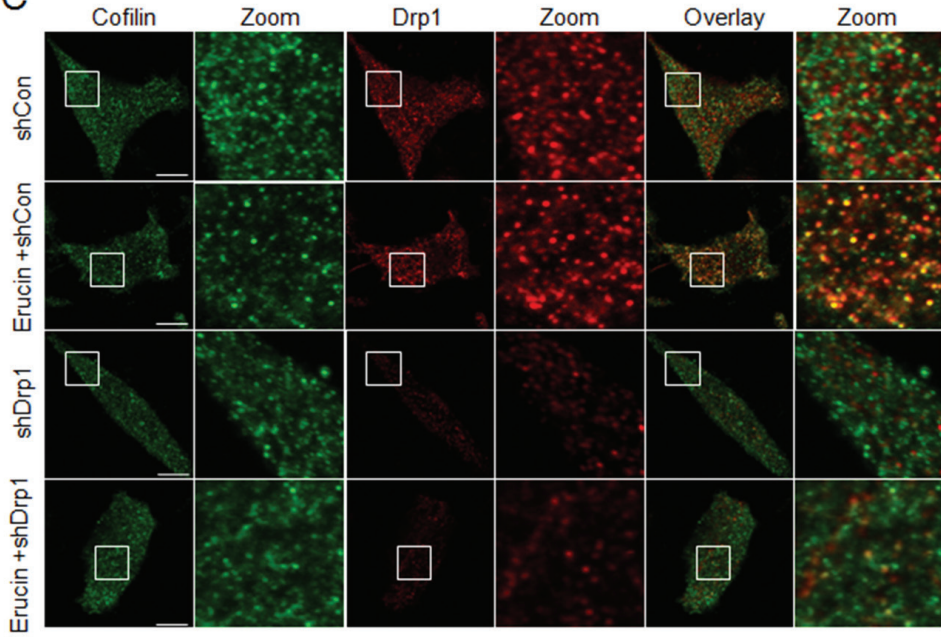

$\mathrm{D}$

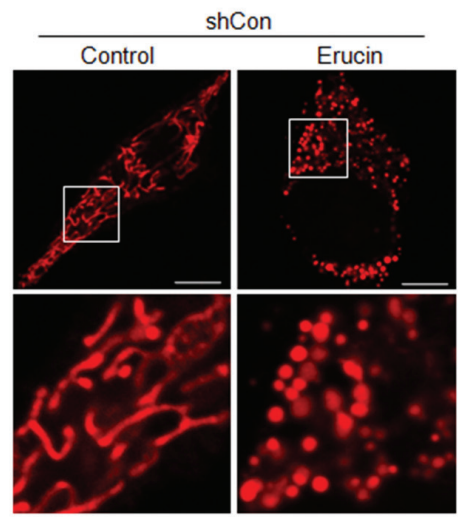

B

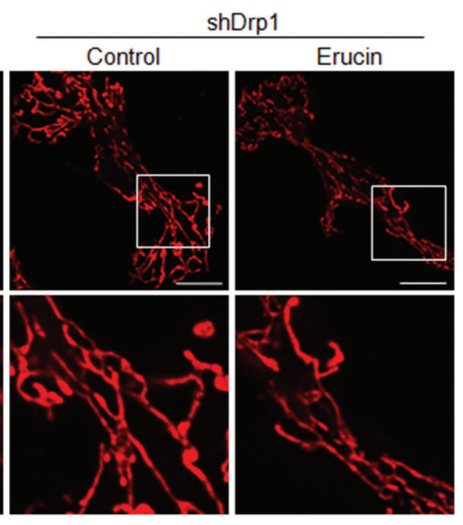

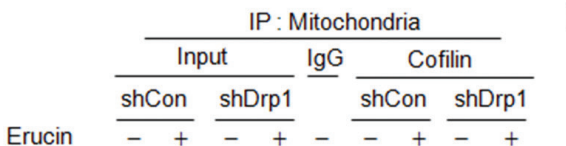

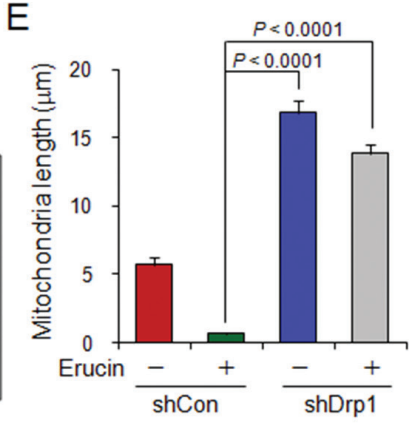

$\mathrm{F}$

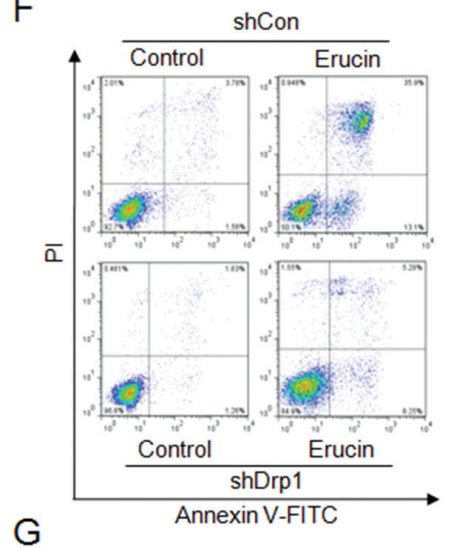

G

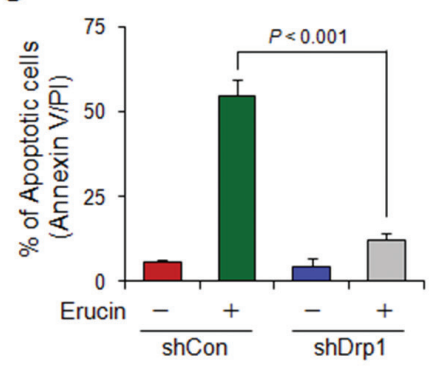

$\mathrm{H}$

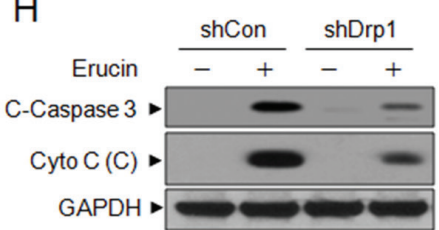

Figure 5: Drp1 knockdown decreases erucin-mediated mitochondrial fission and apoptosis. MDA-MB-231 cells were infected with lentivirus containing constructs that encoded scrambled control shRNA (shCon) or human Drp1-specific shRNA (shDrp1). Stable cell lines were treated without or with $20 \mu \mathrm{M}$ erucin for $6 \mathrm{~h}$. (A) Whole cell lysates (WCL), mitochondrial (Mito) and cytosolic (Cyto) fractions were determined by immunoblotting. (B) Mitochondrial fractions were prepared and subjected to immunoprecipitation to determine the interaction of cofilin and Drp1. (C-E) Cells were immunostained with cofilin (Alexa Fluor 488, green) and Drp1 (Alexa Fluor 647, red) or Mitotracker Red CMXRos. Fluorescence images were collected by confocal microscopy. Scale bar represents $10 \mu \mathrm{m}$. Quantifications of mitochondrial length were performed as described in Methods. (F, G) Apoptosis was measured by flow cytometry. (H) C-Caspase 3 in whole cell lysates and Cyto c in cytosolic fractions (C) were determined by immunoblotting.

localization of cofilin and mitochondrial fission mediated by erucin (Fig. 6B-6D). Furthermore, cofilin S3A significantly enhanced, whereas cofilin S3E reduced, erucin-mediated cytochrome c release, caspase 3 activation and apoptosis (Fig. 6E and 6F). Thus, our data indicated that the dephosphorylation of cofilin (Ser 3) mediated by erucin is required for cofilin translocation to mitochondria and increased mitochondrial fission and apoptosis.

Recent findings have indicated that dephosphorylation of Drp1 at Ser 637 causes Drp1 translocation to mitochondria and increases mitochondrial fission $[26,30]$. Phosphorylation of Drp1 at Ser 616 by Cdk1/Cyclin B promotes mitochondrial fission during mitosis [31]. We then 


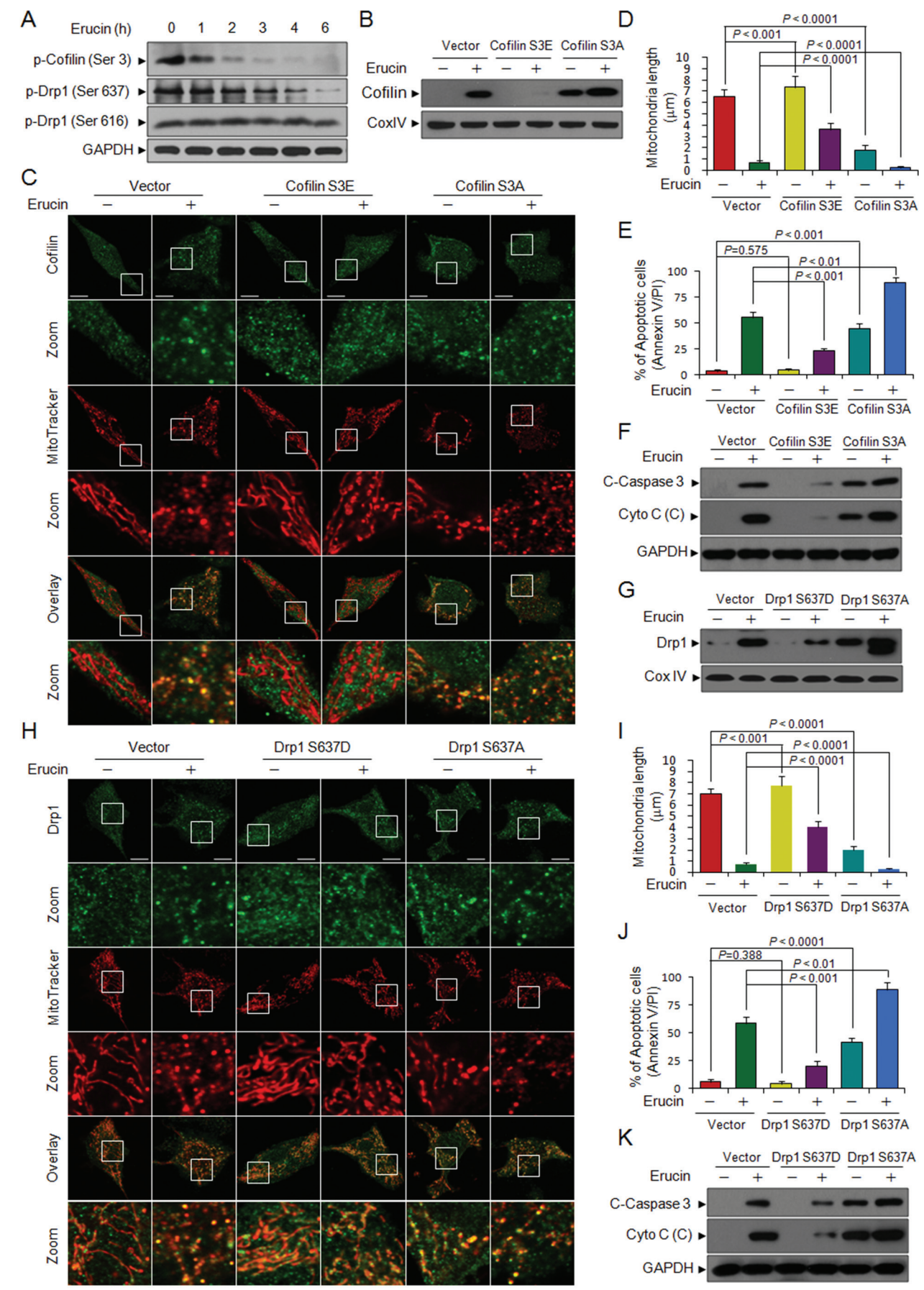

Figure 6: Dephosphorylation of cofilin (Ser 3) and Drp1 (Ser 637) is required for erucin-induced mitochondrial fission and apoptosis. (A) MDA-MB-231 cells were treated with $20 \mu \mathrm{M}$ erucin, whole cell lysates were determined by immunoblotting. (B) MDA-MB-231 cells were transfected with control empty vectors or human cofilin dephosphorylated (active, S3A) mutant plasmids or pseudophosphorylated (inactive, S3E) mutant plasmids for $48 \mathrm{~h}$. After treatment with $20 \mu \mathrm{M}$ erucin for $6 \mathrm{~h}$, mitochondrial fractions were determined by immunoblotting. (C, D) Cells were double-stained with Mitotracker Red CMXRos and cofilin (Alexa Fluor 488, green). Fluorescence images were collected by confocal microscopy. Scale bar represents $10 \mu \mathrm{m}$. Mitochondrial length was measured as described. (E) Apoptosis was measured by flow cytometry. (F) C-Caspase 3 in whole cell lysates and Cyto c in cytosolic fractions (C) were determined by immunoblotting. (G) MDA-MB-231 cells were transfected with control empty vectors or human Drp1 dephosphorylated (active, S637A) mutant plasmids or pseudophosphorylated (inactive, S637D) mutant plasmids for $48 \mathrm{~h}$. After treatment with $20 \mu \mathrm{M}$ erucin for $6 \mathrm{~h}$, mitochondrial fractions were determined by immunoblotting. (H, I) Confocal microscope images of transfected cells labeled with MitoTracker Red CMXRos and Drp1 (Alexa Fluor 647, green). Scale bar represents $10 \mu \mathrm{m}$. Mitochondrial length was measured as described. (J) Apoptosis was measured by flow cytometry. (K) C-Caspase 3 in whole cell lysates and Cyto $\mathrm{c}$ in cytosolic fractions (C) were determined by immunoblotting. 
examined the effect of erucin on phosphorylation of Drp1 at Ser 637 and Ser 616. Exposure of cells to erucin resulted in significantly decrease in the levels of phospho-Drp1 at Ser 637 , but had no effect on phosphorylation of Drp1 at Ser 616 (Fig. 6A). To further examine the role of Drp1 Ser 637 dephosphorylation in erucin-induced mitochondrial fission and apoptosis, we generated a mutant of Drp1 Ser 637D (S637D) to mimic the constitutively phosphorylated form or a mutant of Drp1 Ser 637A (S637A) to mimic the dephosphorylated form. Overexpression of Drp1 S637A significantly increased, whereas Drp1 S637D decreased, the mitochondrial translocation/localization of Drp1 and mitochondrial fission mediated by erucin (Fig. 6G-6I). Moreover, Drp1 S637A significantly increased, whereas Drp1 S637D reduced, erucin-mediated cytochrome c release, caspase 3 activation and apoptosis (Fig. 6J and 6K). These findings indicated that dephosphorylation of Drp1 Ser 637 is required for erucin-induced mitochondrial translocation of Drp1, mitochondrial fission and apoptosis.

\section{ROCK1 is involved in erucin-induced dephosphorylation and mitochondrial translocation of cofilin and Drp1, mitochondrial fission, and apoptosis}

It has been shown that cofilin can be dephosphorylated by the phosphatases PP1 and PP2A, which are regulated by the ROCK1 signaling pathway $[17,32-35]$, and phosphorylated by LIM kinase, which is also regulated by ROCK1 [36-38]. ROCK1 is involved in the regulation of Drp1 phosphorylation status and promote Drp1 translocation to mitochondria, leading to mitochondrial fission [19]. We next examined whether erucin affects the expression of ROCK1, PP1, PP2A, LIMK1, LIMK2 and phospho-LIMK1(Thr508)/ LIMK2(Thr505). Treating cells with erucin resulted in a marked decrease in the levels of ROCK1 and increased cleavage of ROCK1 in a time-dependent manner (Fig. 7A). Exposure of cells to erucin also resulted in marked increase in levels of PP1 and PP2A in time-dependent manners. However, the levels of LIMK1, LIMK2 and phosphoLIMK1(Thr508)/LIMK2(Thr505) were not altered by erucin treatment (Supplementary Fig. S1).

To further assess the functional significance of ROCK1 activation in regulating the phosphorylation status and mitochondrial translocation of cofilin and Drp1, a ROCK1 inhibitor Y-27632 was employed. Pretreating cells with Y-27632 significantly decreased erucin-mediated ROCK1 cleavage/activation and dephosphorylation of cofilin (Ser 3) and Drp1 (Ser 637) (Fig. 7B). Pretreatment with Y-27632 also markedly decreased erucin-mediated translocation of cofilin and Drp1 from the cytosol to mitochondria (Fig. 7C). Y-27632 also significantly decreased erucin-induced mitochondrial fission (Fig. 7D and 7E). Moreover, Y-27632 significantly attenuated erucin-mediated cytochrome c release, caspase 3 activation and apoptosis (Fig. $7 \mathrm{~F}$ and $7 \mathrm{G}$ ).

To further confirm these results, a lentivirus shRNA approach was used to stably knockdown ROCK1 expression. Knockdown of ROCK1 significantly blocked erucin-mediated dephosphorylation of cofilin (Ser 3) and Drp1 (Ser 637) (Fig. 7H), Knockdown of ROCK1 also blocked erucin-mediated translocation of cofilin and Drp1 from the cytosol to mitochondria (Fig. 7I). ROCK1 suppression by shRNA significantly inhibited erucin-mediated mitochondrial fission (Fig. 7J and 7K). Furthermore, depletion of ROCK1 also significantly decreased erucin-mediated cytochrome c release, caspase 3 activation and apoptosis (Fig. 7L and 7M). Taken together, these findings indicated that activation of ROCK1 played a critical role in erucin-mediated dephosphorylation and mitochondrial translocation of cofilin and Drp1, mitochondrial fission, and apoptosis.

\section{Erucin inhibits tumor growth in a MDA-MB-231 xenograft mouse model}

To determine whether our in vitro findings could be replicated in vivo, nude mice were inoculated subcutaneously with MDA-MB-231 cells followed by injections with vehicle or erucin $(50 \mathrm{mg} / \mathrm{kg}$, i.p.) for 50 days starting one week after tumor inoculation. Treatment with erucin resulted in a significant increase in survival compared with untreated controls $(P<0.001)$ (Fig. 8A). Treatment with erucin resulted in a significant suppression of tumor growth after 3 weeks of drug exposure $(P<0.01$ or $P<0.0001$ vs vehicle control) (Fig. $8 \mathrm{~B}$ ). However, there were no significant changes in body weight (Fig. $8 \mathrm{C}$ ) or other signs of potential toxicity such as agitation, impaired movement and posture, indigestion or diarrhea.

To evaluate the effect of erucin on mitochondrial dynamics in the MDA-MB-231 xenograft in vivo, we used immunofluorescence microscopy to examine the morphology of mitochondria in primary cells isolated from the MDA-MB-231 xenograft in vehicle control or erucin-treated mice. Following treatment with erucin, mitochondria became short and fragmented, as evidenced by a significant decrease in the average length of mitochondria (Fig. 8D and 8E). Moreover, apoptosis and Western blot analyses showed that treatment with erucin significantly increased cytochrome c release, caspase 3 activation and apoptosis in primary cells isolated from the MDA-MB-231 xenograft (Fig. 8F and 8G).

Western blot analysis was used to further evaluate whether mitochondrial translocation of cofilin and Drp1 occurred during erucin-mediated mitochondrial fission and apoptosis in primary cells. Cofilin and Drp1 were found in mitochondrial fractions following treatment with erucin, concomitant with the disappearance of cofilin and Drp1 from cytosolic fractions (Fig. 8G). Thus, our data indicated that mitochondrial translocation of cofilin 

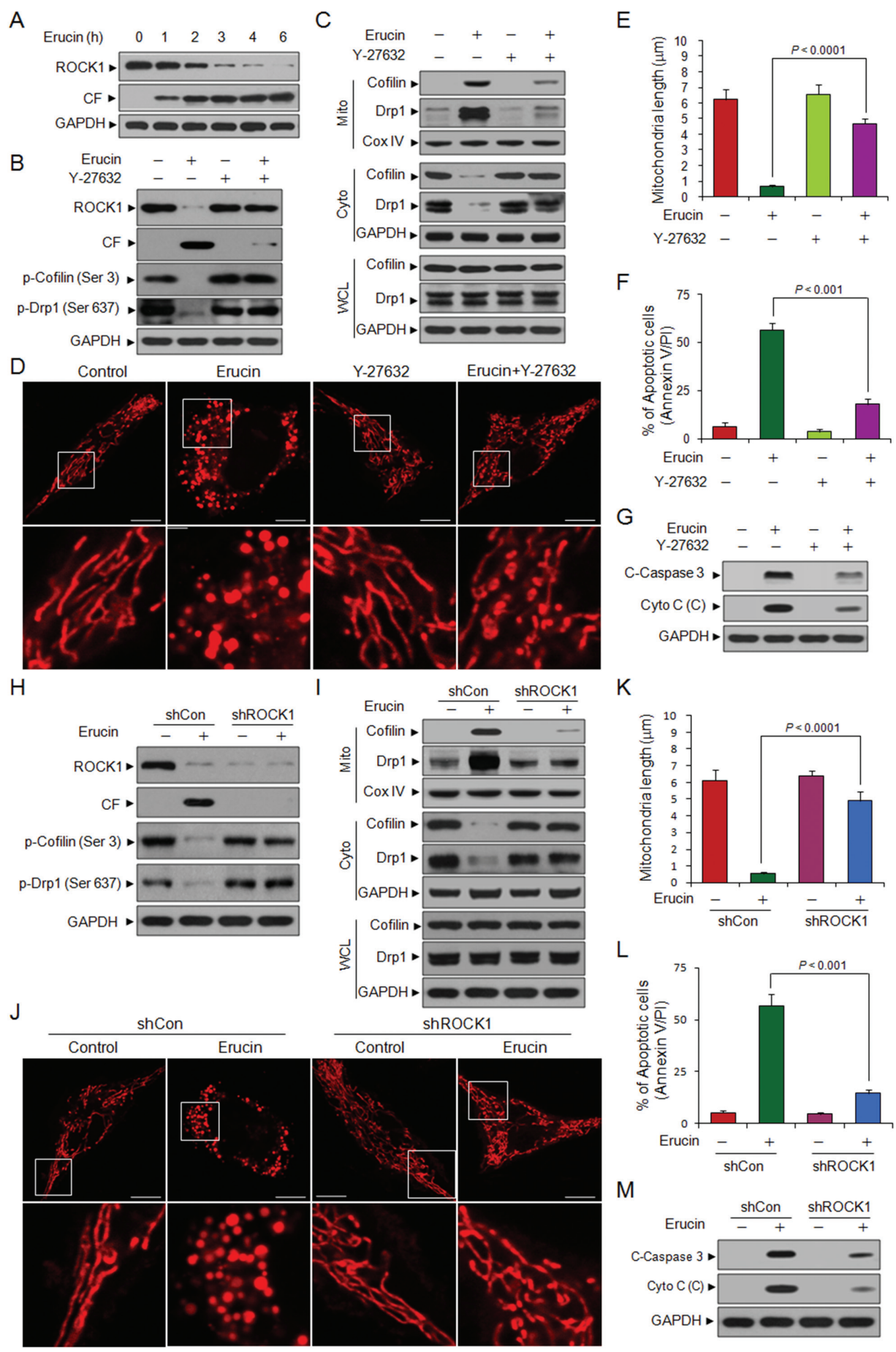

Figure 7: Inhibition of ROCK1 activation or knockdown of ROCK1 attenuate erucin-induced cofilin and Drp1 dependent mitochondrial fission and apoptosis. (A) MDA-MB-231 cells were treated with $20 \mu \mathrm{M}$ erucin, whole cell lysates were determined by immunoblotting. (B, C) MDA-MB-231 cells were pretreated with $20 \mu \mathrm{M} \mathrm{Y}-27632$ for $2 \mathrm{~h}$, followed by treatment with $20 \mu \mathrm{M}$ erucin for $6 \mathrm{~h}$. Whole cell lysates (WCL), mitochondrial (Mito) and cytosolic (Cyto) fractions were determined by immunoblotting. CF represents cleavage fragment. (D, E) Cells were stained with Mitotracker Red CMXRos and observed by confocal microscope. Scale bar represents $10 \mu \mathrm{m}$. Mitochondrial length was measured as described. (F) Apoptosis was measured by flow cytometry. (G) Whole cell lysates and cytosolic fractions were determined by immunoblotting. (H, I) MDA-MB-231 cells were infected with lentivirus containing constructs that encoded scrambled control shRNA (shCon) or human ROCK1-specific shRNA (shROCK1). Stable cell lines were treated without or with $20 \mu \mathrm{M}$ erucin for $6 \mathrm{~h}$. Whole cell lysates (WCL), mitochondrial (Mito) and cytosolic (Cyto) fractions were probed by immunoblotting. (J, K) Cells were stained with Mitotracker Red CMXRos and observed by confocal microscope. Mitochondrial length was measured as described. Scale bar represents $10 \mu \mathrm{m}$. (L) Apoptosis was measured by flow cytometry. (M) Whole cell lysates and cytosolic fractions were determined by immunoblotting. 
A

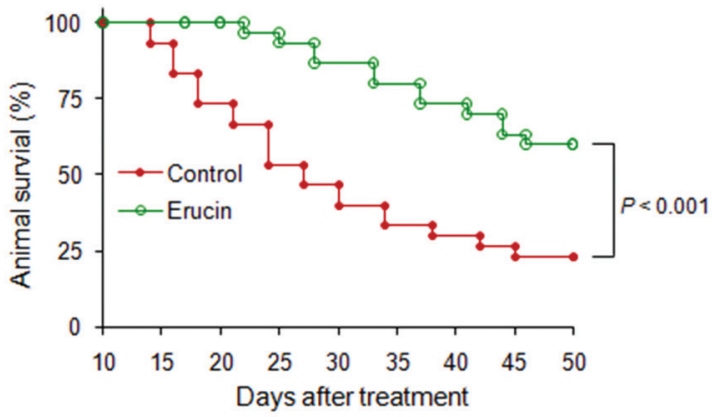

C
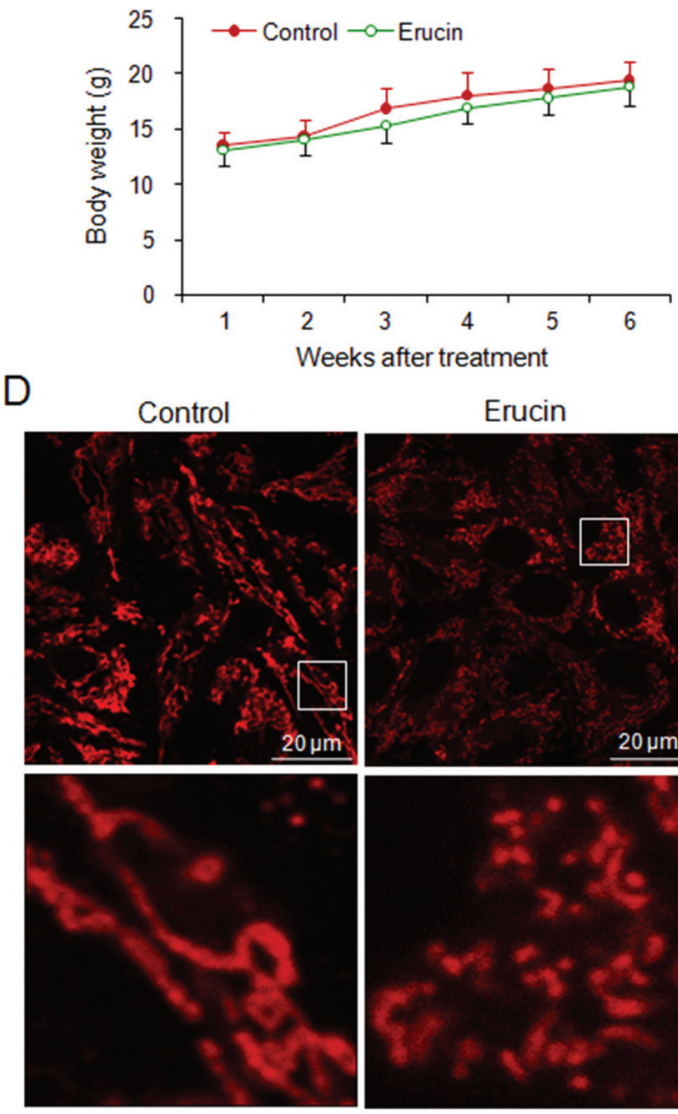
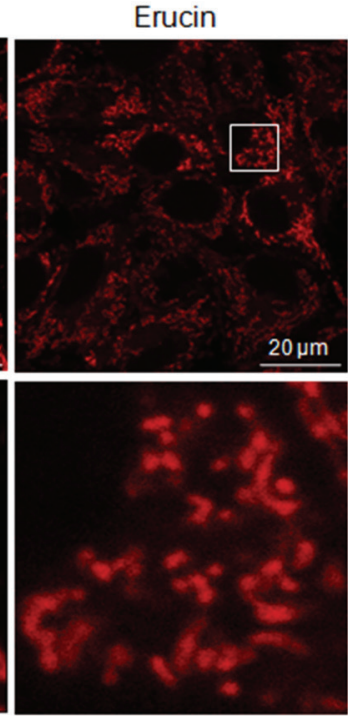

B
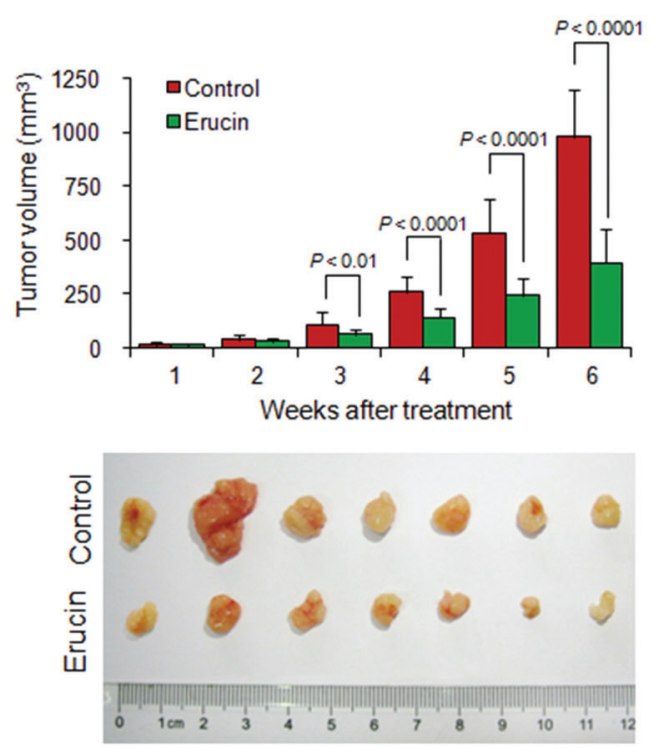

E

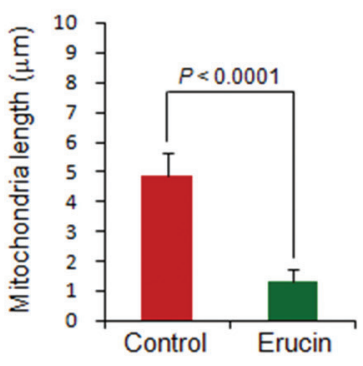

$\mathrm{F}$

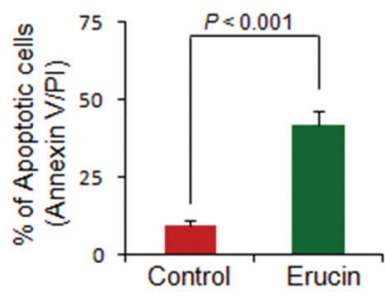

G
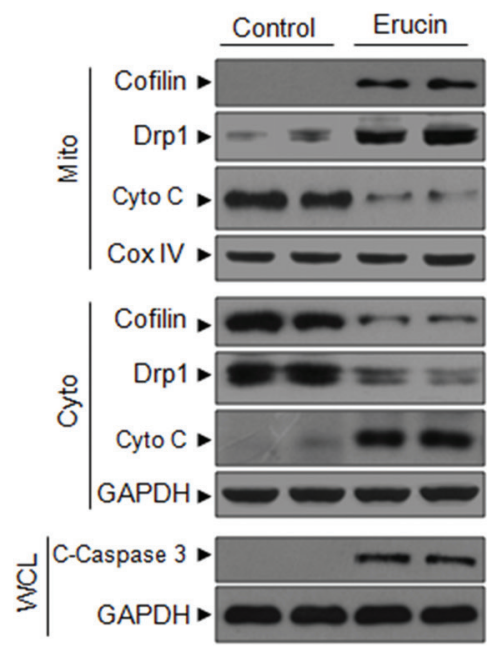

Figure 8: Erucin inhibits tumor growth of MDA-MB-231 xenograft model. (A) The comparison of animal survival between erucin-treated group and vehicle-treated group. $p$-values were calculated using Kaplan-Meier method. (B) Average tumor volume in vehicle control mice and mice treated with erucin. $p<0.01$ or $p<0.0001$ compared with vehicle control. (C) Body weight changes of mice during the 50 days of erucin treatment. (D, E) Primary cells were isolated from representative tumor tissues and cultured in DMEM medium and stained with Mitotracker Red CMXRos. Fluorescence images were collected by confocal microscopy. Mitochondrial length was measured as described. Scale bar represents $20 \mu \mathrm{m}$. (F) Apoptosis of primary cells was measured by flow cytometry. (G) Whole cell lysates (WCL), mitochondrial (Mito) and cytosolic (Cyto) fractions from representative tumor tissues in two vehicle-treated mice and two erucin-treated mice were prepared and subjected to Western blot analysis.

and Drp1 occurred during erucin-induced mitochondrial fission and apoptosis in vivo.

\section{DISCUSSION}

The results of the present study demonstrated for the first time that erucin potently induced apoptosis by triggering mitochondrial fission and that this phenomenon was due primarily to the interaction and recruitment of cofilin and Drp1 to mitochondria. Cofilin is a member of the ADF/cofilin family, which regulates actin dynamics by increasing the rate of actin depolymerization [11]. Recent evidence has demonstrated that mitochondrial translocation of cofilin induces the release of cytochrome $\mathrm{c}$ and apoptosis in neutrophils and neuroblastoma and lymphoma cells $[12,39]$. However, the mechanism by which cofilin 
affects mitochondrial injury remains elusive. Our data showed that erucin-mediated mitochondrial fission and apoptosis was accompanied by mitochondrial translocation of cofilin. Knockdown of cofilin markedly reduced erucinmediated mitochondrial fission and apoptosis. Thus, these data suggest that mitochondrial translocation of cofilin is crucial for erucin-mediated mitochondrial fission and apoptosis.

The most interesting finding of the present study was that the interaction and colocalization of cofilin and Drp1 at mitochondria was involved in erucin-mediated mitochondrial fission during apoptosis. The dynamin-related protein Drp1 is essential for most types of mitochondrial fission [26, 40]. Drp1 is a cytosolic GTPase that has been proposed to couple GTP hydrolysis to membrane constriction and fission [41]. Recent evidence has revealed that Drp1 translocates to mitochondria and mediates mitochondrial fission [42]. Drp1 homo-oligomerizes and forms a ring around the mitochondrial tubule [43]. Drp1 complexes might generate mechanical force via conformational changes, leading to membrane constriction and fission [44]. The role of cofilin in erucin-mediated Drp1 recruitment and mitochondrial fission is strongly supported by several lines of evidence. First, both cofilin and Drp1 translocated from the cytosol to mitochondria during erucin-induced mitochondrial fission. Second, cofilin and Drp1 interacted and colocalized at the outer mitochondrial membrane during erucin-mediated mitochondrial fission. Third, knockdown of cofilin or Drp1 significantly blocked the interaction and colocalization between cofilin and Drp1, mitochondrial fission and apoptosis. To the best of our knowledge, this is the first report to demonstrate that the interaction and mitochondrial translocation of cofilin and Drp1 are required for erucinmediated mitochondrial fission and apoptosis.

Here, we report that erucin-induced mitochondrial fission depends on the phosphorylation status of cofilin and Drp1. The regulation of cofilin involves the phosphorylation and dephosphorylation of its Ser 3 residue; cofilin is inhibited by phosphorylation [45] and translocates to mitochondria only in its dephosphorylated (active) form [12]. The dephosphorylated form of cofilin (S3A) promotes oxidant-mediated mitochondrial damage and apoptosis [39]. Consistent with these findings, our data indicate that only dephosphorylated cofilin translocates to mitochondria. Interestingly, we found that constitutively active cofilin S3A (dephosphorylated) enhanced, whereas the dominantnegative cofilin S3E (phosphorylated) blocked, the mitochondrial translocation of cofilin, mitochondrial fission and apoptosis induced by erucin. Thus, erucin-mediated dephosphorylation of cofilin at Ser 3 is required for the translocation of cofilin to mitochondria and increased mitochondrial fission and apoptosis.

The dynamin-related protein Drp1 is essential for most types of mitochondrial fission $[4,26]$. The majority of the available evidence has revealed that regulation of Drp1 by post-translational modifications is important for
Drp1 translocation to mitochondria $[46,47]$. It has been reported that calcineurin-dependent dephosphorylation of Drp1 at Ser 637 regulates its translocation to mitochondria and subsequent mitochondrial fission [48]. The increased dephosphorylation of Drp1 at Ser 637 regulates its translocation to mitochondria and induces mitochondrial fission, which leads to an increased response to apoptotic stimuli [49-51]. However, another report demonstrated that phosphorylation of Drp1 Ser 600 (corresponding to Ser 637 in human) of mouse podocytes promotes Drp1 translocation to mitochondria and increases mitochondrial fragmentation [19]. Our findings demonstrate that dephosphorylation of Drp1 at Ser 637 regulates its translocation to mitochondria and induces mitochondrial fission in response to erucin based on the following evidence. First, erucin induced dephosphorylation of Drp1 at Ser 637 but not Ser 616. Second, overexpression of Drp1 mutant S637A (dephosphomimetic) increased, whereas Drp1 mutant S637D (phosphomimetic) reduced, the mitochondrial translocation of Drp1, fission and apoptosis. Thus, dephosphorylation of Drp1 at Ser 637 by erucin is required for the translocation of Drp1 to mitochondria and increased mitochondrial fission and apoptosis.

The present study demonstrated that activation of ROCK1 was essential for dephosphorylation of cofilin (Ser 3) and Drp1 (Ser 637), which promoted cofilin and Drp1 recruitment to mitochondria and mitochondrial fission. Recent studies have shown that ROCK1 plays an important role in regulation of apoptosis in various cell types $[16,17]$. ROCK1 has diverse functional activities in different cell types $[18,37]$. ROCK1 activation can regulate activation/dephosphorylation of cofilin by induction of PP1 and PP2A phosphatase activities [17, 32], or control phosphorylation of cofilin by LIM kinase [36-38]. Our data indicate that erucin-induced ROCK1 activation and subsequent activation/dephosphorylation of cofilin are mainly caused by increasing the PP1 and PP2A phosphatase activities. The LIM kinase seems not to be involved in this process. It has been shown that ROCK1 plays an important role in remodeling mitochondrial morphology by regulating the phosphorylation and mitochondria translocation of Drp1 in podocytes [19]. Our previous study showed that ROCK1 activation plays a critical role in regulating cofilin mitochondrial translocation and apoptosis in leukemia cells [17]. Our findings suggest that activation of ROCK1 plays a critical role in erucin-mediated dephosphorylation and mitochondrial translocation of cofilin and Drp1, mitochondrial fission and apoptosis, based on the following evidence: (i) Erucin induced activation of ROCK1 in a time-dependent manner; (ii) Inhibition of ROCK1 activity by Y-27632 or knockdown of ROCK1 by shRNA attenuated erucin-mediated cofilin and Drp1 dephosphorylation and mitochondrial translocation; (iii) Inhibition of ROCK1 activity by Y-27632 or 
knockdown of ROCK1 by shRNA significantly reduced erucin-induced mitochondrial fission and apoptosis.

In summary, the present findings demonstrate for the first time that erucin induces mitochondrial fission and apoptosis in breast cancer cells through mitochondrial translocation and interaction of cofilin and Drp1. Collectively, these observations suggest a hierarchy of events in erucin-induced apoptosis in which ROCK 1 activation represents the primary event, leading to the dephosphorylation of cofilin (Ser 3) and Drp1 (Ser 637), which are translocated from the cytosol to the mitochondria, culminating in mitochondrial fission, cytochrome c release and apoptosis.

\section{MATERIALS AND METHODS}

\section{Antibodies and chemicals}

The following antibodies were used for Western blot analysis: Drp1 (5391, 1:1,000 working dilution), cleaved-caspase 3 (9661, 1:1,000), cleaved-caspase 9 (9505, 1:1,000), Cox IV (4850, 1:2,000), phospho-cofilin (Ser 3) (3313, 1:500), phospho-Drp1 (Ser 637) (4867, 1:500), phospho-Drp1 (Ser 616) (3455, 1:500), LIMK1 (3842, 1:2,000), LIMK2 (3845, 1:1,000) and phosphoLIMK1(Thr508)/LIMK2(Thr505) (3841, 1:500) were purchased from Cell Signaling Technology; GAPDH (sc-25778, 1:500), cytochrome c (sc-13156, 1:1,000), cofilin (sc-376476, 1: 2,000) and PP1 (sc-7482, 1:1,000) were purchased from Santa Cruz Biotechnology; PARP (ab32071, 1:1,000) and ROCK1 (ab45171, 1:1,000) were from Abcam; PP2A (610555, 1:5,000) was purchased from BD Biosciences. Erucin (sc-204741) and Y-27632 (sc216067) were purchased from Santa Cruz Biotechnology.

\section{Cell culture}

MDA-MB-231 and MCF-7 cells were purchased from the American Type Culture Collection, who authenticates cell lines with short tandem repeat profiling and monitoring cell morphology. Cells were maintained in Dulbecco's Modified Eagle Medium (DMEM) containing $10 \% \mathrm{FBS}$ at $37^{\circ} \mathrm{C}$ in $5 \% \mathrm{CO}_{2}$ and passaged for less than 6 months after receipt.

\section{Lentiviral gene transfer and gene silencing}

The human cofilin1 shRNA (5'-CCGGAAGGTGTTCAATGACATGAAACTC GAGTTTCATGTCATTGAACACCTTTTTTTG-3')and human Rock1 shRNA (5'-CCGGGCACCAGTTGTACCCGATTTACTCGAGT AAATCGGGTACAACTGGTGCTTTTTG-3') were synthesized and subcloned into the pLKO.1 plasmid; Human DRP1 shRNA plasmid (sc-43732) and control shRNA plasmid (sc-108060) were purchased from Santa Cruz Biotechnology (Santa Cruz, CA, USA). Plasmids were cotransfected with lentiviral packaging vectors ( $\mathrm{pLP} 1, \mathrm{pLP} 2$ and pLP/VSVG) into 293FT cells using Lipofectamine 3000 according to the manufacturer's instructions. The lentiviruscontaining supernatant was harvested and was used to infect the MDA-MB-231 cells. Cells were subsequently grown under $5 \mu \mathrm{g} / \mathrm{ml}$ puromycin selection to establish stable cell lines.

\section{Site-directed mutagenesis and transfection}

Dephosphorylated (active, S3A) and pseudophosphorylated (inactive, S3E) human cofilin constructs were a gift from Professor James Bamburg (Colorado State University, USA); dephosphorylated (active, S637A) and pseudophosphorylated (inactive, S637D) Drp1 mutants were generated using the QuickChange Site-Directed Mutagenesis Kit (Stratagene, CA, USA) with the following primers: S637A (FW: 5'-AGTTCCTGTTGCACGAAACTAGCTGCTCGGG AAC-3'; RV: 5'-GTTCCCGAGCAGCTAGTTTT CGTGCAACAGGAACT-3'), S637D (FW: 5'-CCAGTTCCTGTTGCACGAAAACTAGATGCTC GGGAACAGCGAGATTGTGAG-3'; RV: 5'-CTCA CAATCTCGCTGTTCCCGAGCATCTAGTTTTCGTG

CAACAGGAACTGG-3'). The mutated Drp1 was confirmed by sequence analysis. Plasmids were transfected into MDAMB-231 cells using Lipofectamine 3000 according to the manufacturer's instructions. After $48 \mathrm{~h}$ of transfection, the cells were exposed $20 \mu \mathrm{M}$ erucin for $6 \mathrm{~h}$ and subsequently subjected to immunoblotting or immunofluorescence analysis.

\section{Apoptosis and mitochondrial membrane potential assay}

Apoptotic cells were detected using the Annexin V-FITC/PI staining kit (PharMingen, San Diego, CA) as previously described [17]. The mitochondrial membrane potential ( $\triangle \Psi \mathrm{m})$ was monitored using 3,3'-dihexyloxacarbocyanine (DiOC, , Molecular Probes), briefly, after treatment, cells were incubated with $40 \mathrm{nM}$ $\mathrm{DiOC}_{6}$ at $37^{\circ} \mathrm{C}$ for 15 minutes, washed twice with PBS and subsequently analyzed by flow cytometry (BectonDickinson, San Jose, CA) with an excitation wavelength of $488 \mathrm{~nm}$ and an emission wavelength of $530 \mathrm{~nm}$ to determine the percentage of cells exhibiting low levels of $\mathrm{DiOC}_{6}$ uptake.

\section{Mitochondrial and cytosolic fraction preparation}

Mitochondrial and cytosolic fractions were obtained as described previously [17].

\section{Immunoprecipitation and western blot analysis}

Cells were lysed in $1 \%$ NP-40 buffer $(50 \mathrm{mM}$ Tris (pH 7.4), $150 \mathrm{mM} \mathrm{NaCl}, 1 \%$ Nonidet P-40, 10\% glycerol, $1 \mathrm{mM}$ PMSF, $10 \mu \mathrm{g} / \mathrm{ml}$ aprotinin, $10 \mu \mathrm{g} / \mathrm{ml}$ leupeptin, $1 \mathrm{mM} \mathrm{Na}_{3} \mathrm{VO}_{4}$ ). Equal quantities of proteins were initially 
precleared by incubation with non-specific normal mouse IgG (Pierce Biotechnology) and protein $\mathrm{A} / \mathrm{G}$ agarose beads (Pierce Biotechnology) for $1 \mathrm{~h}$. After centrifugation, the supernatant was incubated with cofilin antibody overnight at $4^{\circ} \mathrm{C}$. Immunoprecipitates were then collected using protein $\mathrm{A} / \mathrm{G}$ agarose beads followed by several washes in lysis buffer. The samples were separated by SDS-PAGE, transferred to PVDF membranes, and processed for immunoblotting as previously described [17].

\section{Immunofluorescence}

Cells were grown on 12-mm coverslips, stained with 500 nM Mitotracker Red CMXRos (M7512, Molecular Probes) for $30 \mathrm{~min}$ at $37^{\circ} \mathrm{C}$ and washed twice with PBS. The cells were then fixed with $4 \%$ formaldehyde for 15 min, permeabilized with $0.1 \%$ Triton $\mathrm{X}-100$ for $10 \mathrm{~min}$, and blocked with 1\% BSA in PBS for 30 min. Immunostaining was performed using the antibodies including anti-cofilin (1:50, sc-376476, Santa Cruz Biotechnology), antiDrp1 (1:50, 5391, Cell Signaling Technology) and anticytochrome c (1:50, sc-13156, Santa Cruz Biotechnology), followed by the appropriate secondary antibodies Alexa Fluor 488 goat anti-mouse (A11001, Molecular Probes) or Alexa Fluor 647 donkey anti-rabbit (A31573, Molecular Probes), for $1 \mathrm{~h}$ at room temperature. Images were collected using a Leica scanning confocal microscope (TCS SP2 AOB, Wetzlar, Germany). Mitochondrial length was measured as previously described [52]. Briefly, the Mitotracker-stained regions in cells with clearly resolved mitochondria were measured using the line tool in the Leica Application Suite (version: 2.4.1) software. For each group, approximately 100 mitochondria from at least five different cells were counted.

\section{Transmission electron microscopy}

Cells were harvested by trypsinization and fixed with ice-cold 4\% glutaraldehyde in PBS (pH 7.4) at $4{ }^{\circ} \mathrm{C}$ overnight. Next, the cells were postfixed in $2 \%$ osmium tetroxide, dehydrated in a graded series of ethanol, and embedded in Epon 812 resin. Ultra-thin sections $(0.05 \mu \mathrm{m})$ were obtained using glass knives and double-stained with uranyl acetate/lead citrate. Finally, the cells were observed using a Hitachi-7500 electron microscope (Hitachi Instrument, Tokyo, Japan).

\section{Xenograft assay}

Animal studies were performed according to federal guidelines and approved by the Third Military Medical University Institutional Animal Care and Use Committee. Nude mice ( 5 weeks old) were obtained from Vital River Laboratories (Beijing, China). MDA-MB-231 cells $\left(2 \times 10^{6}\right.$ cells/mouse $)$ were suspended in serum-free DMEM and injected subcutaneously into the right flank of each mouse. Mice were randomized into two groups
( $n=30$ per group). One week after tumor inoculation, the mice received either erucin $(50 \mathrm{mg} / \mathrm{kg}$, i.p., five times per week) or an equal volume of vehicle. Signs of potential toxicity, such as agitation, impaired movement and posture, indigestion or diarrhea, and areas of redness or swelling were observed during the treatment. Tumor volume, body weight and survival time were measured at various time intervals throughout the study. Tumor volumes were calculated according to the formula (width ${ }^{2}$ $\times$ length) $/ 2$. Mice were sacrificed at the termination of the experiment, and tumor tissues from representative mice were lysed and subjected to Western blot analysis. Primary cells were isolated from tumor tissues, cultured in DMEM and then subjected to immunofluorescence and apoptosis analysis.

\section{Statistical analysis}

All data values are represented as mean \pm SD. Statistical analysis was performed using two-tailed Student's $t$-tests. Survival analysis in the xenograft experiment was performed using the Kaplan-Meier method, and significance was calculated using the logrank test.

\section{ACKNOWLEDGEMENTS}

We thank Professor James Bamburg (Department of Biochemistry and Molecular Biology, Colorado State University, USA) for providing human cofilin S3A and human cofilin S3E plasmids. This work was funded by the National Natural Science Foundation of China (81402970), the Chongqing Natural Science Foundation (CSTC2013jjB10007), and the Open Project Program of State Key Laboratory of Natural Medicines, China Pharmaceutical University (SKLNMKF201406).

\section{Conflict of interest}

The authors declare no conflict of interest.

\section{REFERENCES}

1. Zick M, Rabl R, Reichert AS. Cristae formation-linking ultrastructure and function of mitochondria. Biochim Biophys Acta. 2009; 1793:5-19.

2. Kuznetsov AV, Margreiter R. Heterogeneity of mitochondria and mitochondrial function within cells as another level of mitochondrial complexity. Int J Mol Sci. 2009; 10:1911-1929.

3. Bossy-Wetzel E, Barsoum MJ, Godzik A, Schwarzenbacher R, Lipton SA. Mitochondrial fission in apoptosis, neurodegeneration and aging. Curr Opin Cell Biol. 2003; 15:706-716.

4. Sheridan C, Martin SJ. Mitochondrial fission/fusion dynamics and apoptosis. Mitochondrion. 2010; 10:640-648. 
5. Youle RJ, Karbowski M. Mitochondrial fission in apoptosis. Nat Rev Mol Cell Biol. 2005; 6:657-663.

6. Karbowski M, Youle RJ. Dynamics of mitochondrial morphology in healthy cells and during apoptosis. Cell Death Differ. 2003; 10:870-880.

7. Wang JX, Li Q, Li PF. Apoptosis repressor with caspase recruitment domain contributes to chemotherapy resistance by abolishing mitochondrial fission mediated by dynaminrelated protein-1. Cancer Res. 2009; 69:492-500.

8. Frank S, Gaume B, Bergmann-Leitner ES, Leitner WW, Robert EG, Catez F, Smith CL, Youle RJ. The role of dynamin-related protein, a mediator of mitochondrial fission, in apoptosis. Dev Cell. 2001; 1:515-525.

9. Estaquier J, Arnoult D. Inhibiting Drp1-mediated mitochondrial fission selectively prevents the release of cytochrome c during apoptosis. Cell Death Differ. 2007; 14:1086-1094.

10. Tanaka A, Youle RJ. A chemical inhibitor of DRP1 uncouples mitochondrial fission and apoptosis. Mol Cell. 2008; 29:409-410.

11. Bernstein BW, Bamburg JR. ADF/cofilin: a functional node in cell biology. Trends Cell Biol. 2010; 20:187-195.

12. Chua BT, Volbracht C, Tan KO, Li R, Yu VC, Li P. Mitochondrial translocation of cofilin is an early step in apoptosis induction. Nat Cell Biol. 2003; 5:1083-1089.

13. Kotiadis VN, Leadsham JE, Bastow EL, Gheeraert A, Whybrew JM, Bard M, Lappalainen P, Gourlay CW. Identification of new surfaces of cofilin that link mitochondrial function to the control of multi-drug resistance. J Cell Sci. 2012; 125:2288-2299.

14. Beck H, Flynn K, Lindenberg KS, Schwarz H, Bradke F, Di Giovanni S, Knoll B. Serum Response Factor (SRF)cofilin-actin signaling axis modulates mitochondrial dynamics. Proc Natl Acad Sci U S A. 2012; 109:E2523-2532.

15. Jacobs M, Hayakawa K, Swenson L, Bellon S, Fleming M, Taslimi P, Doran J. The structure of dimeric ROCK I reveals the mechanism for ligand selectivity. J Biol Chem. 2006; 281:260-268.

16. Coleman ML, Sahai EA, Yeo M, Bosch M, Dewar A, Olson MF. Membrane blebbing during apoptosis results from caspase-mediated activation of ROCK I. Nat Cell Biol. 2001; 3:339-345.

17. Li GB, Cheng Q, Liu L, Zhou T, Shan CY, Hu XY, Zhou J, Liu EH, Li P, Gao N. Mitochondrial translocation of cofilin is required for allyl isothiocyanate-mediated cell death via ROCK1/PTEN/PI3K signaling pathway. Cell communication and signaling : CCS. 2013; 11:50.

18. Julian L, Olson MF. Rho-associated coiled-coil containing kinases (ROCK): Structure, regulation, and functions. Small GTPases. 2014; 5:e29846.

19. Wang W, Wang Y, Long J, Wang J, Haudek SB, Overbeek P, Chang BH, Schumacker PT, Danesh FR. Mitochondrial fission triggered by hyperglycemia is mediated by ROCK1 activation in podocytes and endothelial cells. Cell Metab. 2012; 15:186-200.

20. Melchini A, Traka MH. Biological profile of erucin: a new promising anticancer agent from cruciferous vegetables. Toxins (Basel). 2010; 2:593-612.

21. Jakubikova J, Sedlak J, Mithen R, Bao Y. Role of PI3K/ Akt and MEK/ERK signaling pathways in sulforaphaneand erucin-induced phase II enzymes and MRP2 transcription, G2/M arrest and cell death in Caco-2 cells. Biochem Pharmacol. 2005; 69:1543-1552.

22. Melchini A, Traka MH, Catania S, Miceli N, Taviano MF, Maimone P, Francisco M, Mithen RF, Costa C. Antiproliferative activity of the dietary isothiocyanate erucin, a bioactive compound from cruciferous vegetables, on human prostate cancer cells. Nutr Cancer. 2013; 65:132-138.

23. Abbaoui B, Riedl KM, Ralston RA, Thomas-Ahner JM, Schwartz SJ, Clinton SK, Mortazavi A. Inhibition of bladder cancer by broccoli isothiocyanates sulforaphane and erucin: characterization, metabolism, and interconversion. Mol Nutr Food Res. 2012; 56:1675-1687.

24. Jakubikova J, Bao Y, Sedlak J. Isothiocyanates induce cell cycle arrest, apoptosis and mitochondrial potential depolarization in HL-60 and multidrug-resistant cell lines. Anticancer Res. 2005; 25:3375-3386.

25. Perfettini JL, Roumier T, Kroemer G. Mitochondrial fusion and fission in the control of apoptosis. Trends Cell Biol. 2005; 15:179-183.

26. Knott AB, Perkins G, Schwarzenbacher R, Bossy-Wetzel E. Mitochondrial fragmentation in neurodegeneration. Nat Rev Neurosci. 2008; 9:505-518.

27. Wabnitz GH, Goursot C, Jahraus B, Kirchgessner H, Hellwig A, Klemke M, Konstandin MH, Samstag Y. Mitochondrial translocation of oxidized cofilin induces caspase-independent necrotic-like programmed cell death of T cells. Cell Death Dis. 2010; 1:e58.

28. Roh SE, Woo JA, Lakshmana MK, Uhlar C, Ankala V, Boggess T, Liu T, Hong YH, Mook-Jung I, Kim SJ, Kang DE. Mitochondrial dysfunction and calcium deregulation by the RanBP9-cofilin pathway. FASEB J. 2013; 27:4776-4789.

29. Gu J, Lee CW, Fan Y, Komlos D, Tang X, Sun C, Yu K, Hartzell HC, Chen G, Bamburg JR, Zheng JQ. ADF/ cofilin-mediated actin dynamics regulate AMPA receptor trafficking during synaptic plasticity. Nat Neurosci. 2010; 13:1208-1215.

30. Rambold AS, Kostelecky B, Elia N, Lippincott-Schwartz J. Tubular network formation protects mitochondria from autophagosomal degradation during nutrient starvation. Proc Natl Acad Sci U S A. 2011; 108:10190-10195.

31. Chou CH, Lin CC, Yang MC, Wei CC, Liao HD, Lin RC, Tu WY, Kao TC, Hsu CM, Cheng JT, Chou AK, Lee CI, Loh JK, Howng SL, Hong YR. GSK3beta-mediated Drp1 phosphorylation induced elongated mitochondrial 
morphology against oxidative stress. PLoS One. 2012; 7:e49112.

32. Zhang Y, Kim TH, Niswer L. Phactr4 regulates directional migration of enteric neural crest through PP1, integrin signaling, and cofilin activity. Genes Dev. 2012; 26:69-81.

33. Quintela-Fandino M, Arpaia E, Brenner D, Goh $\mathrm{T}$, Yeung FA, Blaser H, Alexandrova R, Lind EF, Tusche MW, Wakeham A, Ohashi PS, Mak TW. HUNK suppresses metastasis of basal type breast cancers by disrupting the interaction between PP2A and cofilin-1. Proc Natl Acad Sci U S A. 2010; 107:2622-2627.

34. Oleinik NV, Krupenko NI, Krupenko SA. ALDH1L1 inhibits cell motility via dephosphorylation of cofilin by PP1 and PP2A. Oncogene. 2010; 29:6233-6244.

35. Ambach A, Saunus J, Konstandin M, Wesselborg S, Meuer SC, Samstag Y. The serine phosphatases PP1 and PP2A associate with and activate the actin-binding protein cofilin in human T lymphocytes. Eur J Immunol. 2000; 30:3422-3431.

36. Gallo RM, Khan MA, Shi J, Kapur R, Wei L, Bailey JC, Liu J, Brutkiewicz RR. Regulation of the actin cytoskeleton by Rho kinase controls antigen presentation by CD1d. J Immunol. 2012; 189:1689-1698.

37. Riento K, Ridley AJ. Rocks: multifunctional kinases in cell behaviour. Nat Rev Mol Cell Biol. 2003; 4:446-456.

38. Bernard O. Lim kinases, regulators of actin dynamics. Int J Biochem Cell Biol. 2007; 39:1071-1076.

39. Klamt F, Zdanov S, Levine RL, Pariser A, Zhang Y, Zhang B, Yu LR, Veenstra TD, Shacter E. Oxidant-induced apoptosis is mediated by oxidation of the actin-regulatory protein cofilin. Nat Cell Biol. 2009; 11:1241-1246.

40. Otera H, Ishihara N, Mihara K. New insights into the function and regulation of mitochondrial fission. Biochim Biophys Acta. 2013; 1833:1256-1268.

41. Smirnova E, Griparic L, Shurland DL, van der Bliek AM. Dynamin-related protein Drp1 is required for mitochondrial division in mammalian cells. Mol Biol Cell. 2001; 12:2245-2256.

42. Taguchi N, Ishihara N, Jofuku A, Oka T, Mihara K. Mitotic phosphorylation of dynamin-related GTPase Drp1 participates in mitochondrial fission. J Biol Chem. 2007; 282:11521-11529.

43. Okamoto K, Shaw JM. Mitochondrial morphology and dynamics in yeast and multicellular eukaryotes. Annu Rev Genet. 2005; 39:503-536.

44. Danino D, Moon KH, Hinshaw JE. Rapid constriction of lipid bilayers by the mechanochemical enzyme dynamin. J Struct Biol. 2004; 147:259-267.

45. Bamburg JR, Wiggan OP. ADF/cofilin and actin dynamics in disease. Trends Cell Biol. 2002; 12:598-605.

46. Suen DF, Norris KL, Youle RJ. Mitochondrial dynamics and apoptosis. Genes Dev. 2008; 22:1577-1590.

47. Hong Z, Kutty S, Toth PT, Marsboom G, Hammel JM, Chamberlain C, Ryan JJ, Zhang HJ, Sharp WW, Morrow E, Trivedi K, Weir EK, Archer SL. Role of dynamin-related protein 1 (Drp1)-mediated mitochondrial fission in oxygen sensing and constriction of the ductus arteriosus. Circ Res. $2013 ; 112: 802-815$.

48. Cereghetti GM, Stangherlin A, Martins de Brito O, Chang CR, Blackstone C, Bernardi P, Scorrano L. Dephosphorylation by calcineurin regulates translocation of Drp1 to mitochondria. Proc Natl Acad Sci U S A. 2008; 105:15803-15808.

49. Chang CR, Blackstone C. Cyclic AMP-dependent protein kinase phosphorylation of Drp1 regulates its GTPase activity and mitochondrial morphology. J Biol Chem. 2007; 282:21583-21587.

50. Cribbs JT, Strack S. Reversible phosphorylation of Drp1 by cyclic AMP-dependent protein kinase and calcineurin regulates mitochondrial fission and cell death. EMBO Rep. 2007; 8:939-944.

51. Costa V, Giacomello M, Hudec R, Lopreiato R, Ermak G, Lim D, Malorni W, Davies KJ, Carafoli E, Scorrano L. Mitochondrial fission and cristae disruption increase the response of cell models of Huntington's disease to apoptotic stimuli. EMBO Mol Med. 2010; 2:490-503.

52. Korobova F, Ramabhadran V, Higgs HN. An actindependent step in mitochondrial fission mediated by the ER-associated formin INF2. Science. 2013; 339:464-467. 